\title{
What's new in ARDS: ARDS also exists in resource-constrained settings
}

\author{
Elisabeth D. Riviello ${ }^{1,2^{*}}$, Luigi Pisani ${ }^{3,4,5}$ and Marcus J. Schultz ${ }^{5,6,7}$
}

(c) 2016 The Author(s). This article is published with open access at Springerlink.com

The acute respiratory distress syndrome (ARDS) causes morbidity and mortality in both resource-constrained and resource-rich settings, but diagnosis, therapy, and research priorities vary with context (Fig. 1). While ARDS exists in resource-constrained settings, it may be under-recognized and under-treated, and is certainly under-studied. This has consequences both for current ARDS patient in resource-constrained settings, as well as future patients who could benefit from context-specific interventions to improve outcomes in ARDS.

The burden of ARDS in resource-constrained settings is poorly understood. One study recently reported the incidence and outcomes of ARDS in a Rwandan hospital using a modified definition of ARDS [1]. The study suggested that ARDS is both common and frequently lethal in resource-constrained settings: $4 \%$ of adult patients met the modified definition of ARDS, and $50 \%$ of these patients died. A direct comparison between resourceconstrained and resource-rich settings is not possible because of differences in methodology of the available studies [1-3] and predicting where ARDS might be more common is not straightforward. Infection and trauma are the leading clinical insults leading to ARDS, and both are more prevalent in low-income countries [1, 2, 4]. On the other hand, ARDS could be more prevalent in high-income countries given evidence suggesting that mechanical ventilation, far more available in these settings, itself contributes to the development of ARDS [5]. In addition, the distinction between resource-constrained and resource-rich settings is more complicated

\footnotetext{
*Correspondence: beth_riviello@post.harvard.edu

2 Division of Pulmonary, Critical Care and Sleep Medicine, Beth Israel Deaconess Medical Center and Harvard Medical School, 330 Brookline Avenue, Boston, MA 02215, USA

Full author information is available at the end of the article
}

in middle-income countries where resources exist but access to care may be extremely variable [6].

If ARDS is so common and lethal in resource-constrained settings, why is the data so sparse? One reason is a challenge not specific to ARDS but to all critical care research. Critical illness studies are often confined to patients in intensive care units (ICUs), of which there are very few in poorer settings [4]. The recent Intensive Care Over Nations (ICON) study is a good example of a largescale effort to capture an international sample; yet in this study only $1.4 \%$ of all patients were from the African continent [7]. A second reason explaining scarce ARDS data from resource-constrained settings is particular to the current Berlin definition of ARDS, which requires diagnostic and treatment capabilities that are almost universally absent in resource-limited settings [8]. Specifically, arterial blood gas analysis, which is necessary for calculation of the $\mathrm{PaO}_{2}$ to $\mathrm{FiO}_{2}$ ratio $(\mathrm{P} / \mathrm{F})$, and chest radiography, which is necessary to determine whether bilateral opacities are present, are often not accessible [9].

Validation of alternative criteria to the Berlin definition for ARDS could allow for better recognition and quantification of ARDS in resource-constrained settings. Lung ultrasound (US) may be easier to perform than chest radiography in resource-constrained settings, and increasing evidence suggests that lung US is at least as accurate as chest radiography for diagnosing ARDS [10]. Ultrasound can assess positive end-expiratory pressure (PEEP)-induced changes in lung aeration and thus has the potential to guide recruitment maneuvers. Furthermore, combining lung with bedside cardiac US can be helpful in differentiating ARDS from cardiogenic pulmonary edema and in assessing right ventricular function [11]. Advantages of US are many: fast learning curves, low costs, and requiring only basic US technology. Affordable, handheld, battery-driven devices are increasingly available.

\section{Springer}


Reasonable estimates of the $\mathrm{P} / \mathrm{F}$ from $\mathrm{SpO}_{2}$ to $\mathrm{FiO}_{2}$ ratio $(\mathrm{S} / \mathrm{F})$ have been derived from large datasets, such that pulse oximetry could realistically replace oxygenation assessment by arterial blood gases [12]. The Rwandan study mentioned above demonstrated remarkable consistency in ARDS incidence using a variety of $\mathrm{FiO}_{2}$ estimates and S/F cutoffs [1]. Full validation of the Kigali modification of the Berlin definition for ARDS and definitive validation of the S/F would allow comparisons across different settings worldwide. Furthermore, S/F should be investigated as an alternative to $\mathrm{P} / \mathrm{F}$ in the mounting evidence on better outcome prediction using oxygenation data at $24 \mathrm{~h}$ from ARDS diagnosis [13].

Does it matter that ARDS exists in resource-constrained settings but is rarely recognized? Yes. It matters because recognition is necessary to improve outcomes. First, recognition allows implementation of interventions that are clearly feasible in resource-constrained settings, such as conservative fluid management for both prevention and supportive care [14]. Second, recognition allows research into the risks and benefits of applying interventions that are known to be effective in resource-rich settings but could be less safe in settings with fewer trained staff (Fig. 1). For example, proning for severe ARDS is theoretically possible given that it requires no particular technology; however, the ability to perform it safely with the few staff available may be a barrier. A trial in Bangladesh that found possible harm from early enteral feeding in cerebral malaria is a good example of the need to test interventions in both resource-rich and resource-constrained environments, where the risks and benefits may be very different [15]. Third, recognition allows research into ARDS triggers and lung injury pathways that may be different in various contexts and populations. ARDS associated with malaria, HIV, or tuberculosis in Africa may represent a different set of molecular pathways than ARDS associated with community-acquired pneumonia in Europe.

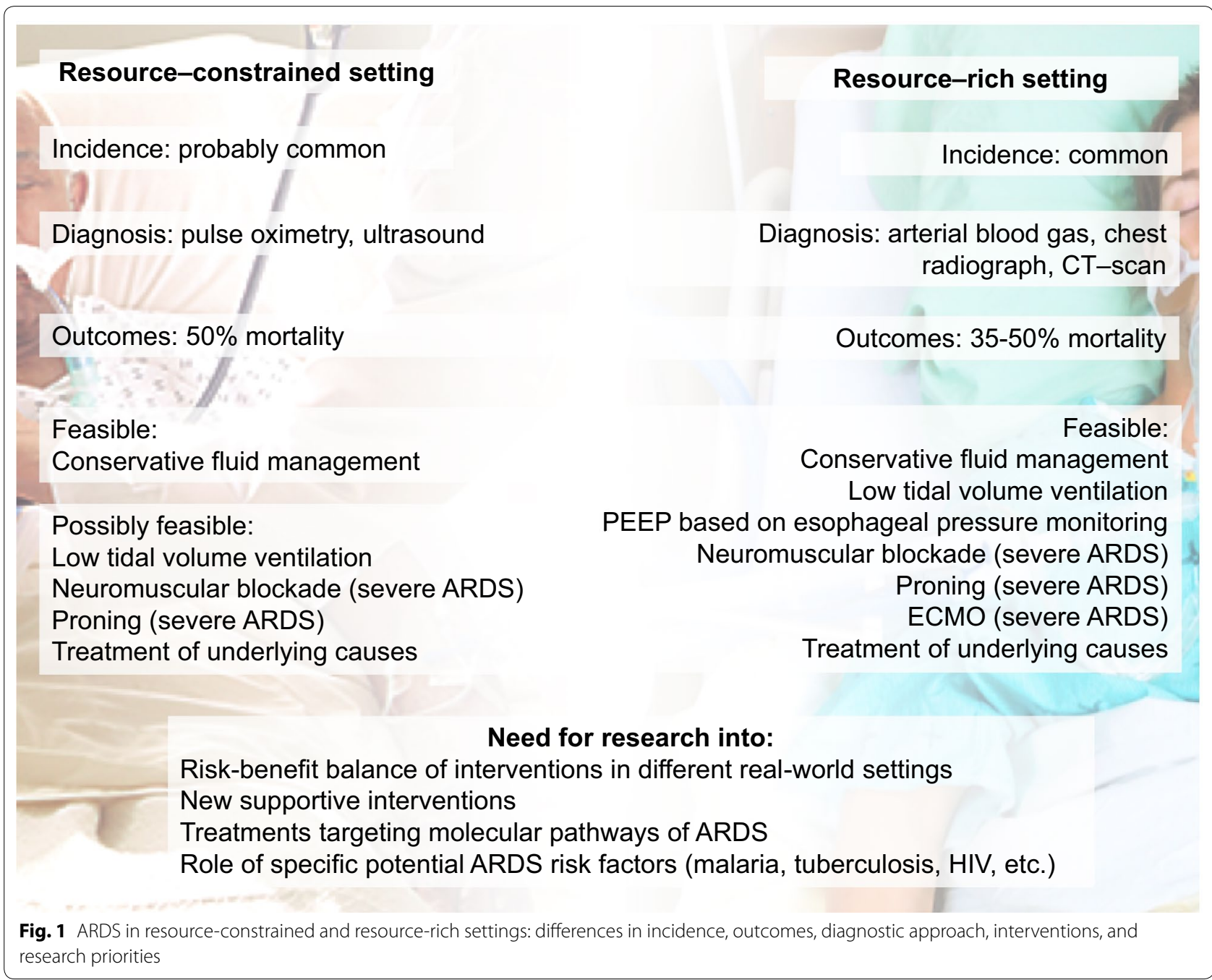


Just as research in sepsis requires one to pursue targeted molecular therapies that include resource-limited settings [16], so too does the heterogeneity of ARDS triggers and presentations highlight the need for trials in settings beyond resource-rich countries. Finally, recognition of ARDS could contribute to improvements in all aspects of healthcare. A good outcome for a patient with ARDS caused by peritonitis requires the capacity for safe surgery and adequate antibiotics in addition to lung-protective ventilation. This sobering limitation is nonetheless a platform for advocacy. Just as a 'vertical' approach to improving HIV outcomes has led to a recognition of a need for 'horizontal' health system strengthening, so too could a focus on ARDS strengthen advocacy for improved healthcare systems.

ARDS exists worldwide, including in resource-poor settings where its incidence, triggers, modifiers, and outcomes remain largely unknown. Validation of a definition that can be applied in all settings, education about how to prevent and manage the syndrome, and research to expand treatment strategies are all desperately needed.

\begin{abstract}
Author details
1 Department of Medicine, University Teaching Hospital of Kigali, Kigali, Rwanda. ${ }^{2}$ Division of Pulmonary, Critical Care and Sleep Medicine, Beth Israel Deaconess Medical Center and Harvard Medical School, 330 Brookline Avenue, Boston, MA 02215, USA. ${ }^{3}$ Department of Emergency and Organ Transplantation, University of Bari 'Aldo Moro', Bari, Italy. ${ }^{4}$ Doctors With AfricaCuamm, Padua, Italy. ${ }^{5}$ Department of Intensive Care, Academic Medical Center, University of Amsterdam, Amsterdam, The Netherlands. ${ }^{6}$ Laboratory of Experimental Intensive Care and Anesthesiology (LEICA), Academic Medical Center, University of Amsterdam, Amsterdam, The Netherlands. ${ }^{7}$ Mahidol-Oxford Research Unit (MORU), Faculty of Tropical Medicine, Mahidol University, Bangkok, Thailand.
\end{abstract}

\section{Open Access}

This article is distributed under the terms of the Creative Commons Attribution-NonCommercial 4.0 International License (http://creativecommons.org/ licenses/by-nc/4.0/), which permits any noncommercial use, distribution, and reproduction in any medium, provided you give appropriate credit to the original author(s) and the source, provide a link to the Creative Commons license, and indicate if changes were made.

Received: 8 February 2016 Accepted: 4 March 2016

Published online: 16 March 2016
References

1. Riviello ED, Kiviri W, Twagirumugabe T et al (2016) Hospital incidence and outcomes of the acute respiratory distress syndrome using the Kigali modification of the Berlin definition. Am J Respir Crit Care Med 193(1):52-59

2. Rubenfeld GD, Caldwell E, Peabody E et al (2005) Incidence and outcomes of acute lung injury. N Engl J Med 353(16):1685-1693

3. Villar J, Blanco J, Anon JM et al (2011) The ALIEN study: incidence and outcome of acute respiratory distress syndrome in the era of lung protective ventilation. Intensive Care Med 37(12):1932-1941

4. Adhikari NK, Fowler RA, Bhagwanjee S, Rubenfeld GD (2010) Critical care and the global burden of critical illness in adults. Lancet 376(9749):1339-1346

5. Villar J, Slutsky AS (2010) Is acute respiratory distress syndrome an iatrogenic disease? Crit Care 14(1):120

6. Austin S, Murthy S, Wunsch H et al (2014) Access to urban acute care services in high- vs. middle-income countries: an analysis of seven cities. Intensive Care Med 40(3):342

7. Vincent JL, Marshall JC, Namendys-Silva SA et al (2014) Assessment of the worldwide burden of critical illness: the Intensive Care Over Nations (ICON) audit. Lancet Respir Med 2(5):380-386

8. Ranieri VM, Rubenfeld GD, Thompson BT et al (2012) Acute respiratory distress syndrome: the Berlin definition. JAMA 307(23):2526-2533

9. Buregeya E, Fowler RA, Talmor DS, Twagirumugabe T, Kiviri W, Riviello ED (2014) Acute respiratory distress syndrome in the global context. Glob Heart 9(3):289-295

10. Volpicelli G, Elbarbary M, Blaivas M et al (2012) International evidencebased recommendations for point-of-care lung ultrasound. Intensive Care Med 38(4):577-591

11. Corradi F, Brusasco C, Pelosi P (2014) Chest ultrasound in acute respiratory distress syndrome. Curr Opin Crit Care 20(1):98-103

12. Brown SM, Grissom CK, Moss M, Rice TW, Schoenfeld D, Hou P, Thompson BT, Brower RG, NIH/NHLBI PETAL Network (2016) Non-linear imputation of $\mathrm{PaO}_{2} / \mathrm{FIO}_{2}$ from $\mathrm{SpO}_{2} / \mathrm{FIO}_{2}$ among patients with acute respiratory distress syndrome. Chest. doi:10.1016/j.chest.2016.01.003

13. Villar J, Fernandez RL, Ambros A et al (2015) A clinical classification of the acute respiratory distress syndrome for predicting outcome and guiding medical therapy. Crit Care Med 43(2):346-353

14. Beitler JR, Schoenfeld DA, Thompson BT (2014) Preventing ARDS: progress, promise, and pitfalls. Chest 146(4):1102-1113

15. Maude RJ, Hoque G, Hasan MU et al (2011) Timing of enteral feeding in cerebral malaria in resource-poor settings: a randomized trial. PLoS One 6(11):e27273

16. Cohen J, Vincent JL, Adhikari NKJ et al (2015) Sepsis: a roadmap for future research. Lancet Infect Dis 15:581-614 